\title{
Morphology of polyamide 6 confined into carbon nanotubes
}

\author{
Agnieszka Piegat ${ }^{1}$, Zygmunt Staniszewski $^{1}$, Artur PoepPel ${ }^{2}$, Miroslawa El Fray $^{1 *}$ \\ ${ }^{1}$ West Pomeranian University of Technology Szczecin, Polymer Institute, Piastow 45, 70-311 Szczecin, Poland \\ ${ }^{2}$ Honda R\&D Europe (Deutschland), Carl-Legien-Str. 30, D-63073 Offenbach/Main, Germany
}

\begin{abstract}
The preparation of polymer nanocomposites filled with carbon nanotubes requires the nanotubes to be uniformly dispersed and compatible with the polymer matrix. In this work we report a preparation method of polyamide 6 (PA 6) based nanocomposite containing multi-walled carbon nanotubes (MWCNT) without any additional surface modification and obtained by in situ polymerization, as a simple method for composites production. The process was assisted by ultrasounds prior to synthesis. With such a method, an interesting morphology of polyamide 6 confined into a multiwalled carbon nanotube as well as grafted on a carbon nanotube surface was observed. For comparative purpose, PA 6 nanocomposites were also prepared from commercially available master batch by melt compounding.
\end{abstract}

Keywords: polyamide 6; carbon nanotubes; in situ polymerization; nanocomposites

(C) Wroclaw University of Technology.

\section{Introduction}

Polymer nanocomposites are important group of modern engineering materials due to their specific properties, such as significantly improved mechanical stability, high-temperature stability, flame retardancy and enhanced barrier properties. These phenomenona are strongly connected to the type of polymer matrix, specific interactions between polymer and nanofiller as well as specific surface area and chemical character of the nanofiller. In recent years nanostructured polymer composites comprising layered silicate clays have been investigated intensively [1-3]. Polyamide/montmoryllonite nanocomposites developed in early 1990s opened the doors to the new and emerging research area, where, using the inherent characteristics of the aluminosilicate layers, such as swelling behavior and cation exchange, the inorganic phase could be evenly dispersed in the polymeric matrix on a nanometer scale, hence, generating strong interactions between the inorganic and organic phases.

In situ intercalative polymerization that guarantees strong polymer-filler interactions was reported

*E-mail: mirfray@zut.edu.pl to be only suitable for clay minerals [1]. However, recently, in situ polymerization was also developed for carbon nanotube-based materials $[4,5]$. Nevertheless, most of the multiwalled carbon nanotubes (MWCNT), eventually can be debonded at the MWCNT-polymer interface and subsequently pulled out from the polymer matrix rather than broken down, indicating rather weak interfacial interaction between the nanotubes and the matrix due to the formation of severe agglomeration of the nanotubes. Therefore, most of the research is focused on interfacial phenomena for CNT-based polymer nanocomposites since they have a strong effect on the mechanical properties. Most of the work in this subject is reported for melt compounded pristine or differently modified nanotubes and polyamide 6 [6-9]. The nanotubes surface functionalization, specifically MWCNT, can be realized by a simple acid treatment in order to obtain MWCNT with different oxygen containing groups on the surface or by more complicated methods, e.g. grafting of other polymers. With this second approach, for example, anionic surface-initiated polymerization of ethylene oxide and styrene has been performed using MWCNT functionalized with anionic initiators [10]. The transmission electron microscopy (TEM) images of polymer grafted 
nanocomposites showed the presence of thick layers of polymer, up to $30 \mathrm{~nm}$, around the MWCNT. However, more interesting strategy towards functional materials would be the synthesis of the systems, where polymers are not only grafted on MWCNT surface but also are confined within the tubes as inorganic/inorganic materials, where polymers are confined within the channels of mesoporous hosts. Among them, in particular composites containing either conducting polymers or metals have been actively investigated as "molecular wires" in electronic devices [10-13]. In such systems, monomers are usually impregnated into mesoporous silicas through adsorption, covalent binding or activated by appropriate initiating species covalently bounded onto pore walls, which are employed subsequently to initiate polymerization. Depending on the monomer type, polymers could be obtained by in situ oxidation polymerization, electropolymerization or coordinative polymerization.

In this work, we present for the first time, the polyamide/CNT nanocomposites with unique morphology, where polyamide chains are not only grafted on MWCNT but also are confined within the nanotubes. The key procedure towards development of this new type morphology was to combine few steps, including ultrasounds treatment of a monomer ( $\varepsilon$-caprolactam), high pressure ring opening polymerization and in situ high temperature polymerization of polyamide 6 in a presence of MWCNT. Importantly, this morphology has been developed during the polyamide-MWCNT synthesis in a semi-pilot plant scale.

\section{Experimental}

\subsection{In situ polymerization of MWCNT- PA 6 nanocomposites}

First, polyamide 6 (PA 6) as the neat (reference) material was synthesized with the laboratory-scale pressure reactor, producing $2.8 \mathrm{~kg}$ of a polymer per batch. Briefly: $3000 \mathrm{~g}$ of $\varepsilon$-caprolactam (kindly supplied by Azoty Tarnow, Poland) was mixed in a closed autoclave for the polymerization. The polymerization temperature was maintained at $260{ }^{\circ} \mathrm{C}$ for $6 \mathrm{~h}$. Flowing nitrogen was used to exhaust water generated during the process. The product was removed from the autoclave and pelletized, extracted in water at $80{ }^{\circ} \mathrm{C}$ for $12 \mathrm{~h}$, and then dried in a vacuum oven for $12 \mathrm{~h}$. The MFR (Melt Flow Rate) of synthesized PA $6\left(2.16 \mathrm{~kg}, 230{ }^{\circ} \mathrm{C}\right)$ was $26.5 \mathrm{~g} / 10 \mathrm{~min}$.

MWCNT-PA 6-based nanocomposite was obtained by in situ polymerization. In detail: firstly, $30 \mathrm{~g}$ of MWCNT (NC7000, Nanocyl), $2000 \mathrm{~g}$ $\varepsilon$-caprolactam, and $100 \mathrm{~g}$ distilled water were portioned, ultrasonicated for $30 \mathrm{~min}$ and homogenized with high speed stirrer (20000 rpm) to form a master solution. Then, the master solution and additional $1000 \mathrm{~g} \varepsilon$-caprolactam were mixed in a closed autoclave under 11 bar pressure. Next, the process was continued according the procedure as described for PA 6 synthesis. In this way, nanocomposites containing $1 \mathrm{wt} . \%$ of MWCNT was prepared.

For comparative purpose, PA 6 nanocomposites were also prepared from commercially available master batch (PlastiCyl PA1503, composed of PA 6 and 15 wt.\% NC7000, Nanocyl). Melt compounding of the neat PA 6 with PlastiCyl PA1503 master batch was performed with a use of twin screw extruder at the temperature range from 180 to $230{ }^{\circ} \mathrm{C}$ at $125 \mathrm{rpm}$ for $20 \mathrm{~min}$. Final CNT content was 1 wt. $\%$ and 2 wt. $\%$, respectively.

\subsection{Scanning electron microscopy (SEM)}

The morphologies of MWCNT-PA 6 nanocomposites were observed on cryofractured surfaces with the use of the JEOL JSM-6100 scanning electron microscope at $20 \mathrm{kV}$ acceleration voltages. Before observations, the samples were coated with gold/palladium nanolayer.

\subsection{Transmission electron microscopy (TEM)}

A transmission electron microscope FEI Tecnai F20 with a $200 \mathrm{kV}$ acceleration voltage was used to study the MWCNT-PA 6 nanocomposites morphology. The specimens for TEM observations were cut from the bulk material at room temperature using a Leica Ultramicrotome. Ultrathin sections, approximately $50 \mathrm{~nm}$ thick, were 
cut using a diamond knife and were collected on 300 mesh copper grids.

For MWCNT observations a droplet of the $3 \mathrm{wt} . \%$ of MWCNT suspension in ethylene glycol (Nanocyl) was placed on a 300 mesh copper grid. Subsequently, ethylene glycol was evaporated over 24 hours in order to obtain dry powder on the grid surface.

\section{Results and discussion}

TEM micrograph of the MWCNT powder with a typical tubular structure with open ends is presented in Fig. 1a. Dimensions were determined by measuring 10 randomly chosen nanotubes. The outer tube diameter is around $14 \mathrm{~nm}$, the inner diameter is around $5 \mathrm{~nm}$ (Fig. 1b). The nanotubes are of very good quality, without any contaminations from catalyst or graphite.

It is well-known that uniform dispersion of a filler in the polymer matrix is required to improve mechanical as well as physical properties of composite materials. Broza et al. [14] demonstrated a significant influence of oxidized single wall carbon nanotubes on the increase of Young's modulus, tensile strength, and strain to failure at a very low amount, from 0.01 to $0.1 \mathrm{wt} . \%$, of CNTs in the PBT matrix. Zhou and Burkhart [15] presented strong relationship between nanofiller hydrophobicity and interactions with the polymer matrix. After modification of hydrophilic silica with different alkyl chain lengths, both storage modulus and loss modulus of all $\mathrm{iPP} / \mathrm{SiO}_{2}$ nanocomposites significantly increased. Such strong enhancement of the properties is directly related to nanofiller-polymer matrix interactions. Therefore, to investigate the interactions between MWCNT and PA 6, the morphology of MWCNT-PA 6 nanocomposites prepared by in situ process and by the melt compounding was evaluated and compared.

Fracture surface morphology of PA 6 composites containing $1 \mathrm{wt} . \%$ MWCNT prepared by the melt compounding method is shown in Fig. 2a. SEM micrograph reveals a very good distribution of MWCNT in a polymer matrix. Moreover, many little bulges are observed in Fig. 2a, suggesting that

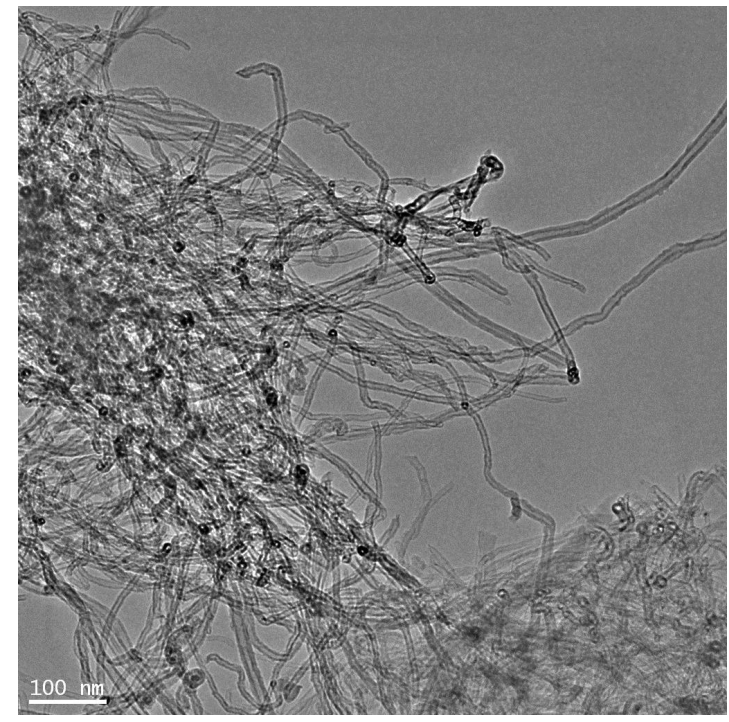

(a)

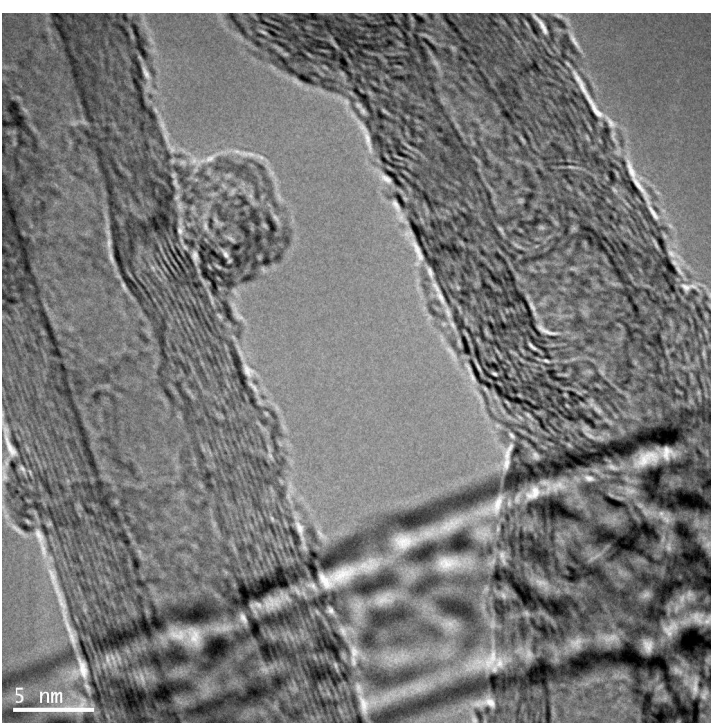

(b)

Fig. 1. TEM micrographs of MWCNT at lower (a) and higher (b) magnifications.

the nanotubes do not break when the cold fracture takes place and they remain in the polymer matrix. Such result suggests that interfacial adhesion between nanofiller and the polymer matrix is strong and no pull-out of MWCNT from polymer matrix occurs. These observations correlate excellently with those presented by Logakis et al. [16], where also masterbatch dilution technique was applied in order to obtain MWCNT-PA 6 composites at much 
higher MWCNT concentration, up to 20 wt.\%. TEM micrograph (Fig. 2b) confirms the uniform dispersion of CNT and also shows their random orientation in the PA 6 matrix. Interestingly, the revealed carbon nanotubes are short (much shorter than pristine CNT presented in Fig. 1a).

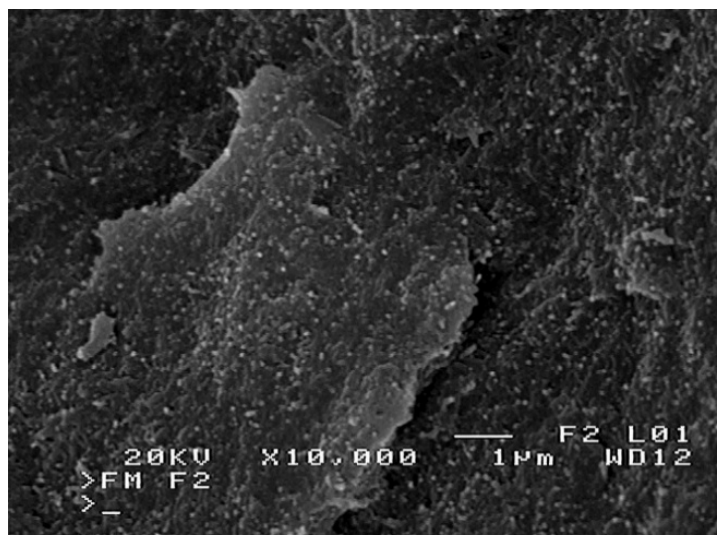

(a)

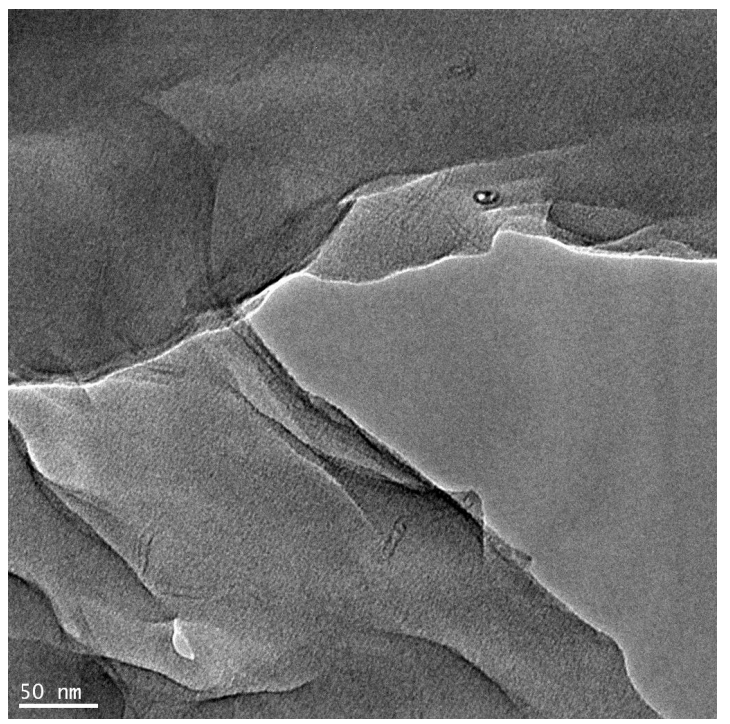

(b)

Fig. 2. SEM image (a) of MWCNT-PA 6 nanocomposite containing $1 \mathrm{wt} . \%$ of nanofiller, produced by melt compounding, (b) TEM image of an ultrathin section.

Recently, it has been demonstrated that in situ methods are preferred for nanocomposites preparation to obtain materials with a uniform distribution of a nanofiller in a polymer matrix [17-19]. Fracture surface and ultrathin section morphologies of MWCNT-PA 6 materials are presented in Fig. 3a, 3b. It can be noticed that carbon nanotubes are well dispersed in the polymer matrix, with many long and individual carbon nanotubes (Fig. 3a). No cavities are present on the fracture surface. The preserved CNT morphology is revealed in TEM micrograph in Fig. 3b illustrating long, individual nanotubes in a polymer matrix. This type of morphology is different from that one observed for materials prepared by melt compounding, where many short CNT, probably defected during high shear forces applied at master batch and nanocomposite preparation, were detected.

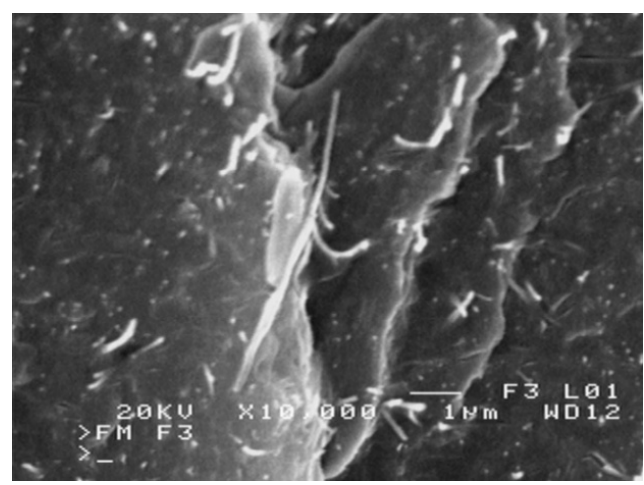

(a)

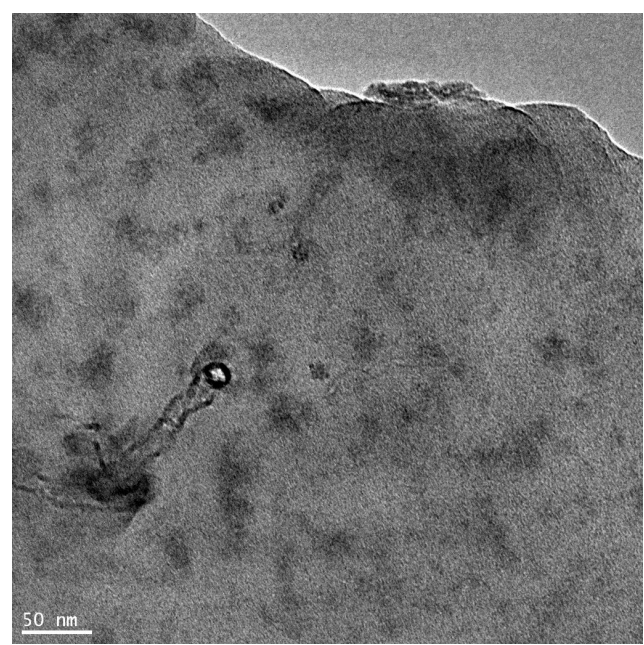

(b)

Fig. 3. SEM image (a) of MWCNT-PA 6 nanocomposite containing $1 \mathrm{wt} \%$ of nanofiller, produced by in situ polymerization, (b) TEM image of an ultrathin section.

At higher magnification (Fig. 4), we can observe much more interesting details regarding 
nanocomposites morphology after in situ polymerization. Specifically, as can be seen from Fig. 4, a nanoscale alignment of the macromolecule chains inside the "host" channel of carbon nanotubes resembles a "molecular wire" as in organic-inorganic systems, however, those were based on mesoporous hosts $[10,12,13]$.

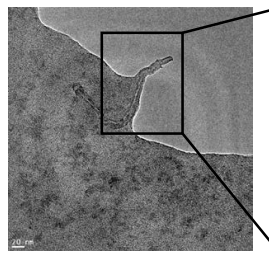

(a)

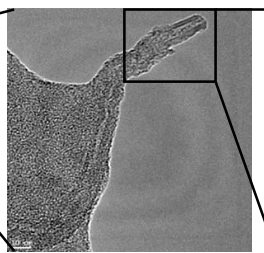

(b)

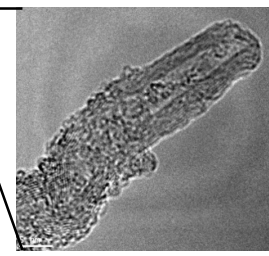

(c)
Fig. 4. TEM image of PA 6-1 wt.\% CNT nanocomposite produced by in situ synthesis, at different magnifications.

In our system, where we used high pressure to initiate polymer chain growth at simultaneous presence of CNT, PA 6 is confined within the carbon nanotube (Fig. 4), and also behaves as a support on which polymer is grafted. This unique morphology was observed for PA 6-based carbon nanotube materials for the first time. Such observations suggest very good wettability of CNT by the monomer at the initial stage of the reaction and possibility of polymerization inside the carbon nanotubes, thus, producing such confined morphology. This mechanism of reaction is also possible from the spatial point of view, in Warshel projection [22]. The maximum diameter of $\varepsilon$-caprolactam is around $0.8 \mathrm{~nm}$ and its height is $0.6 \mathrm{~nm}$, therefore, there is no space hindrance for CNT (5 nm diameter) to be penetrated by the monomer. Importantly, this penetration process occurs without any specific modification of CNT surface.

\section{Conclusions}

In this work, the morphology of MWCNTcontaining PA 6 nanocomposites was investigated. Materials were prepared by conventional melt blending with the use of PA 6 master batch using the in situ polymerization technique. Good dispersion of MWCNT in a polymer

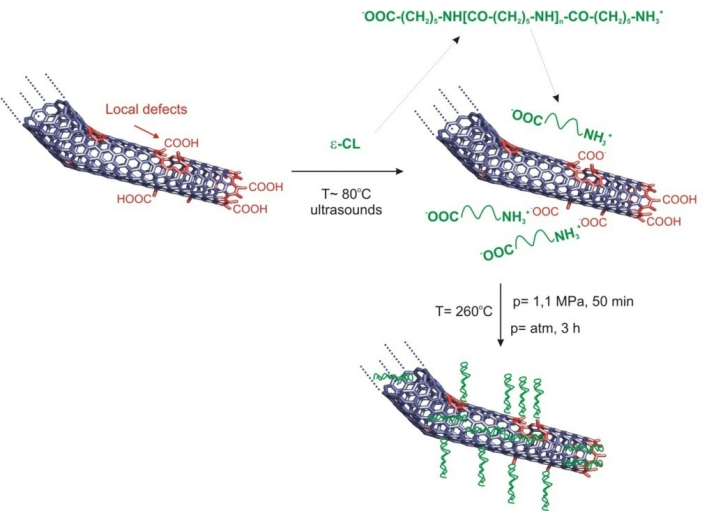

Fig. 5. Graphical scheme for resulted CNT-PA 6 morphology of nanocomposites.

matrix was found for both techniques. We demonstrated that the nanocomposite prepared by in situ polymerization of $\varepsilon$-caprolactam in a presence of $1 \mathrm{wt} . \%$ MWCNT is capable to form interesting morphology, where the polymer is confined in a multiwalled carbon nanotube as well as is grafted on .a nanotube surface (Fig. 5).

\section{References}

[1] Bitinis N., Hernandez M., Verdejo R., Kenny J.M., Lopez-Manchado M.A., Adv. Mater. Sci., 23 (2011), 5229.

[2] Jordan J., Jacob K.I., Tannenbaum R., Sharaf M.A., JASIUK I., Mater. Sci. Eng. A-Struct., 393 (2005), 1.

[3] Jancar J., Douglas J.F., Starr F.W., Kumar S.K., Cassagnau P., Lesser A.J., Sternstein S.S., Buehler M.J., Polymer, 51 (2010), 3321.

[4] Zhang W.L., Shen L., Phang I.Y., LiU T., Macromolecules, 37 (2004), 256.

[5] Vankayala R.R., Lai W.I.P., Cheng K.C., HWang K.C., Polymer, 52 (2011), 3337.

[6] Li J., Fang Z., Tong L., Gu A., LiU F., Eur. Polym. J., 42 (2006), 3230.

[7] Logakis E., Pandis C., Peoglos V., Pissis P., PiONTECK J., PÖtschKe P., MičUŠíK M., OMAstova M., Polymer, 50 (2009), 5103.

[8] Schartel B., Pötschke P., Knoll U., AbdelGoAD M., Eur. Polym. J., 41 (2005), 1061.

[9] Kodgire P.V., Bhattacharyya A.R., Bose S., Gupta N., Kulkarni N.R., Misra A., Chem. Phys. Lett., 432 (2006) 480.

[10] Xu L., Ye Z., Cui Q., Gu Z., Mercier L., Polymer, 52 (2011), 5961.

[11] Li Y., Shimizu H., J. Polym. Sci. A1, 44 (2005), 284.

[12] Kimura M., Iwashima Y., Ohta K., Hanabusa K., ShIRAi H., Macromolecules, 38 (2005), 5055. 
[13] Lin V.S.Y., RAdU D.R., HaN M.K., Deng W., Kuroki S., Shanks B.H., Pruski M., J. Am. Chem. Soc., 124 (2002), 9040.

[14] Broza G., Kwiatkowska M., RosŁaniec Z., SChulte K., Polymer, 46 (2005), 5860.

[15] Zhou R.J., Burkhart T., J. Mater. Sci., 46 (2011), 1228.

[16] Logakis E., Pandis C., Peoglos V., Pissis P., Stergiou C., Pionteck J., PÖtschKe P., MiČUŠíK M., Omastova M., J. Polym. Sci., 47 (2009), 764.

[17] Kim S.H., PARK S.H., Kim S.C., Polym. Bull., 53 (2005), 285.

[18] Chang J.H., UK AN Y., Ryu S., Giannelis E.P., Polym. Bull., 51 (2003), 69.

[19] El Fray M., Rybko M., Piegat A., Mater. Sci. Forum, 714 (2012), 277.
[20] Kumar S., DANG T.D., ARnOld F.E., BhatTACHARYya A.R., Min B.G., Zhang X., VAiA R.A., Park C., Adams W.W., Hauge R.H., Smalley R.E., RAMESH S., WILlis P.A., Macromolecules, 35 (2002), 9039.

[21] Deng J., Ding X., Zhang W., Peng Y., Wang Y., Long X., Li P., Chan A.S.C., Eur. Polym. J., 38 (2002), 2497.

[22] Warshel A., Levitt M., Livson S., J. Mol. Spectr., 33 (1970), 84.

Received 2014-06-12

Accepted 2015-01-29 